\title{
Effects of the Sport Health-Related Fitness Program on Muscular Endurance, Flexibility, Cardiovascular Endurance, and Muscular Strength and Power in Korean College Students
}

\author{
Eun-Hye KIM ${ }^{1}$, *Wi-Young SO ${ }^{2}$ \\ 1. Department of Physical Education, Korea University, Seoul, Korea \\ 2. Sports and Health Care Major, Korea National University of Transportation, Chungju-si, Korea \\ *Corresponding Author: Email: wowso@ut.ac.kr
}

(Received 09 Mar 2018; accepted 22 Mar 2018)

\section{Dear Editor-in-Chief}

The percentage of Korean college students who participate in some form of physical activity three times a week is only $28.4 \%$. Adults over the age of $20 \mathrm{yr}$ are the only group that participates in regular exercise on a less frequent basis $(22.8 \%)$ than Korean college students (1). Due to time constraints related to their studies and job searches, the amount of time that these students have for physical activity is estimated to be about 18 min per week (2). In line with global increases in obesity rates among people in their 20s (3), the obesity rate among Korean college students rose dramatically from $15.2 \%$ in 1998 to $23.9 \%$ in 2014, which is a significant increase compared to other age groups (4). Decreased participation in physical activity results in deteriorating physical fitness and an increased risk of obesity and other metabolic syndrome risk factors (5-6). Therefore, to improve college students' physical fitness levels, health-related fitness programs are required.

The purpose of the health-related fitness program is not only to improve physical fitness during the college years but also to develop lifelong exercise and physical fitness habits. College students, however, often view exercise as strenuous and tiresome because of the perception that repetition and overload training are required. Therefore, the health-related fitness program has adopted the New Sports method to circumvent these negative impressions and promote positive attitudes towards exercise and physical fitness. This study aimed to verify the effectiveness of the 'Sport Health-Related Fitness' programs, such as New Sports, in improving college students' physical fitness.

Overall, 1614 college freshmen (941 men and 673 women) who took the 'Sport Health-Related Fitness' program at Daejeon University, Daejeon-si, Korea from 2013 to 2015 participated in the study. The participants underwent health-related fitness measurements before and after participating in the course, which required $100 \mathrm{~min}$ of physical fitness activity per week for $14 \mathrm{wk}$. The 'Sport Health-Related Fitness' Program is described in Table 1.

Informed consent was obtained from each student prior to their participation in the study. Based on the American College of Sports Medicine (7) and Ministry of Culture, Sports and Tourism of Korea (8), the health-related fitness test consisted of factors that evaluated muscular endurance, flexibility, cardiovascular endurance, and muscular strength and power.

The data were analyzed with SPSS Ver. 18.0 (SPSS, Chicago, IL, USA), used to calculate the means and standard deviations. Paired t-tests were used to analyze the effectiveness of the 'Sport Health-Related Fitness' program. Statistical significance was set at $P<0.05$. After the 14 week 'Sport Health-Related Fitness' program, a 
significant improvement was seen in muscular endurance, flexibility, cardiovascular endurance, and $50 \mathrm{~m}$ muscular strength and power in both men and women $(P<0.001)$ (Table 2$)$.

Table 1: ‘Sport Health-Related Fitness’ program description

\begin{tabular}{|c|c|c|c|}
\hline Week & Level & $\begin{array}{c}\text { Time } \\
\text { (minutes) }\end{array}$ & Content \\
\hline & Warm-up & 10 & $400 \mathrm{~m}$ walking $1 \mathrm{set}$ \\
\hline & & 10 & Light stretching, 1 set \\
\hline \multirow[t]{3}{*}{$3-8$} & Main exercise & 30 & Squat, lunge, side flank, knee push-up, sit-ups burpee \\
\hline & & 10 & Break time \\
\hline & & 40 & Dodge ball, flying- disc, handler, kinball, floorball, teeball \\
\hline \multirow[t]{3}{*}{$9-14$} & & 30 & Run: $400 \mathrm{~m}$, step-ladder, mini hurdles, rubber cone \\
\hline & & 10 & Break time \\
\hline & & 40 & Squat, lunge, side flank, knee push-up, sit-ups burpee \\
\hline \multirow{4}{*}{15} & & 30 & Run: cycle ergometer, treadmill \\
\hline & & 10 & Break time \\
\hline & & 40 & $\begin{array}{l}\text { Back extension, bench-press, pull down, leg extension, leg curl, leg press, pec- } \\
\text { deck flyer, arm curl, hack squat }\end{array}$ \\
\hline & Cool-down & 10 & Light stretching, 1 set \\
\hline
\end{tabular}

The 'Sport Health-Related Fitness' program improved the health-related fitness of college students in their 20s. Thus, development and distri- bution of these types of exercise programs are needed to enhance the health and wellness of students during their college years and beyond.

Table 2: Changes in muscular endurance, flexibility, cardiorespiratory endurance, and muscular strength and power after 14 wk of the 'Sport Health-Related Fitness' program

\begin{tabular}{|c|c|c|c|c|c|}
\hline Variables & Group & Pre & Post & $\bar{t}$ & $P$ \\
\hline Muscular endurance: & Total & $34.06 \pm 13.87$ & $38.03 \pm 13.17$ & -20.842 & $<0.001^{* * 1 k}$ \\
\hline \multirow[t]{2}{*}{ Sit-ups(reps/min) } & Men & $40.45 \pm 11.32$ & $44.39 \pm 10.50$ & -15.933 & $<0.001^{* 1 * k}$ \\
\hline & Women & $25.13 \pm 12.04$ & $29.14 \pm 11.24$ & -13.429 & $<0.001^{* 1 \times k}$ \\
\hline Flexibility: & Total & $1208 \pm 9.22$ & $14.39 \pm 8.33$ & -19.234 & $<0.001^{* 1 \times k}$ \\
\hline \multirow[t]{2}{*}{ Sit and $\operatorname{reach}(\mathrm{cm})$} & Men & $10.01 \pm 9.14$ & $12.12 \pm 823$ & -12931 & $<0.001^{* 1 \times k}$ \\
\hline & Women & $14.99 \pm 8.54$ & $17.63 \pm 7.27$ & -14.974 & $<0.001^{* * 1 *}$ \\
\hline Cardiorespiratory endurance: & Total & $40.36 \pm 17.85$ & $45.34 \pm 17.58$ & -19.825 & $<0.001^{* * 1 k}$ \\
\hline \multirow[t]{2}{*}{ PACER(reps) } & Men & $50.76 \pm 15.47$ & $55.83 \pm 14.43$ & -13290 & $<0.001^{* * 1 k}$ \\
\hline & Women & $25.83 \pm 8.18$ & $30.66 \pm 8.96$ & -17.419 & $<0.001^{* * 1 *}$ \\
\hline Muscular strength and Power: & Total & $8.74 \pm 1.50$ & $8.39 \pm 1.27$ & 16.438 & $<0.001^{* * *}$ \\
\hline \multirow[t]{2}{*}{$50 \mathrm{~m}$ dash(sec) } & Men & $7.84 \pm 1.05$ & $7.55 \pm 0.70$ & 10.178 & $<0.001^{* * *}$ \\
\hline & Women & $9.99 \pm 1.09$ & $9.56 \pm 0.91$ & 13.653 & $<0.001^{* 1 k *}$ \\
\hline
\end{tabular}

Total $=1,614$; men=941; women $=673$

Data are presented as means \pm standard deviations

$* * * P<0.001$; tested using paired $t$-tests

\section{Conflict of interest}

The authors declare that there is no conflict of interests.

\section{References}

1. Ministry of Culture, Sports and Tourism of Ko- rea (2011). 2010 Korean National Survey on Citizen's sports participation. Korea National Statistics.

2. Ministry of Health and Welfare of Korea \& Korea Centers for Disease Control \& Prevention (2011). Korea Health Statistics 2010: Korea National Health and Nutrition Examination Survey (KNHANES V-1). Korea National Statistics. 
3. Ng M, Fleming T, Robinson M et al (2014). Global, regional, and national prevalence of overweight and obesity in children and adults during 1980-2013: a systematic analysis for the Global Burden of Disease Study 2013. Lancet, 384:766-81.

4. Ministry of Health and Welfare of Korea \& Korea Centers for Disease Control \& Prevention (2015). Korea Health Statistics 2014: Korea National Health and Nutrition Examination Survey (KNHANES VI-2). Korea National Statistics.

5. Healy GN, Wijndaele K, Dunstan DW et al (2008). Objectively measured sedentary time, physical activity, and metabolic risk: the Australian Diabetes, Obesity, and Lifestyle Study (AusDiab). Diabetes Care, 31 (2):369-371.

6. Bauman AE (2004). Updating the evidence that physical activity is good for health: an epidemiological review 2000-2003. J Sci Med Sport, 7(1 Suppl):6-19.

7. American College of Sports Medicine (2006). ACSM's guidelines for exercise testing and prescription. Philadelphia: Lippincott Williams \& Wilkins.

8. Ministry of Culture, Sports and Tourism of Korea (2015). 2015 The Survey of National Physical Fitness. Korea National Statistics. 